\title{
INTRA VITREAL TRIAMCINOLONE ACETONIDE FOR DIFFUSE DIABETIC MACULAR OEDEMA
}

\author{
B. Koshalram¹, N. Sivakumar², S. Venkatesh ${ }^{3}$, Anusha $^{4}$ \\ 1 Vitreo Retinal Surgeon, Retina Centre of Coimbatore. \\ ${ }^{2}$ Associate Professor, Department of Ophthalmology, Government Dharmapuri Medical College, Dharmapuri, Tamilnadu. \\ 3 Phaco Surgeon, Jothi Laser Centre, Dharmapuri. \\ ${ }^{4}$ Vitreo Retinal Surgeon, Retina Centre of Coimbatore.
}

ABSTRACT

\section{BACKGROUND}

Diabetic retinopathy is the leading cause of blindness. Diabetic macular oedema is the main reason for the reduced vision.

\section{MATERIALS AND METHODS}

A prospective study of 30 cases of Diffuse Diabetic Macular Oedema (DDME) was undertaken. All eyes identified with DDME were given Intra vitreal Triamcinolone acetonide injection $2 \mathrm{mg}(0.5 \mathrm{ml})$. Changes in visual outcome and central macular thickness were observed over the period of 6 months.

\section{RESULTS}

There was 23.3\% reduction in Central Foveal Thickness (CFT) in 3 weeks post intervention compared to baseline. CFT remains similar at 2 months. Best Corrected Visual Acuity (BCVA) improved one line in 17 eyes (56.6\%) and 2 lines improved in 4 eyes (13.3\%) 3 weeks post intervention. After 2 months BCVA one line in 22 eyes (74.3\%). After 4 months BCVA improved one line in 23 eyes $(76.7 \%)$.

\section{CONCLUSION}

There was statistically significant difference in the central foveal thickness and also statistically significant improvement in visual acuity.

\section{KEYWORDS}

Diabetic Macular Oedema, Triamcinolone Acetonide, Central Foveal Thickness.

HOW TO CITE THIS ARTICLE: Koshalram B, Sivakumar N, Venkatesh S, et al. Intra vitreal triamcinolone acetonide for diffuse diabetic macular oedema. J. Evolution Med. Dent. Sci. 2017;6(9):716-720, DOI: 10.14260/Jemds/2017/154

\section{BACKGROUND}

Diabetic retinopathy is the leading cause of blindness in patients aged 20-74 years. The global prevalence of diabetes is estimated to exceed 220 million people by the year 2001 and 300 million by the year 2025.1 Prevalence rate of diabetes in Indian population ranges from 4-11.6\%. ${ }^{1}$ Macular oedema affects approximately 29 percent of diabetic patients with disease duration of 20 years or more and is the main reason of reduced vision in this population. In the Early Treatment Diabetic Retinopathy (ETDRS) Study 2, the threeyear risk of moderate visual loss (A doubling of the initial visual angle or a decrease of three lines or more on a logarithmic visual acuity chart) for diabetic patients with clinically significant macular oedema (CSME) was 30 percent. In the ETDRS study, 2 laser photocoagulation reduced the risk of moderate visual acuity loss for all eyes with diabetic macular oedema and mild to moderate non-proliferative diabetic retinopathy by about 50 percent. $^{2}$

Financial or Other, Competing Interest: None.

Submission 09-12-2016, Peer Review 15-01-2017,

Acceptance 21-01-2017, Published 30-01-2017.

Corresponding Author:

Dr. N. Sivakumar,

Associate Professor,

Department of Ophthalmology,

Government Dharmapuri Medical College,

Dharmapuri-636701,

Tamilnadu.

E-mail: nskeyedr@yahoo.com

DOI: $10.14260 /$ jemds $/ 2017 / 154$
However, 12 percent of the treated eyes still lost 15 or more ETDRS letters at the three-year follow-up interval. Furthermore, less than 3 percent of treated eyes demonstrated an improvement in visual acuity of the same magnitude.

\section{Aim}

To study the change in visual Outcome and study the serial change in macular thickness as measured by Spectral Domain Optical Coherence Tomography for centre involved diabetic macular oedema when treated with intra vitreal triamcinolone acetonide

\section{MATERIALS AND METHODS}

A prospective study of 30 cases of diffuse Diabetic Macular oedema was undertaken over period of 3 years (January 2012 to January 2015) in Retina Centre of Coimbatore. Most of the cases are referred from ophthalmologists from Dharmapuri and Coimbatore Districts.

All eyes identified as centre involved Diabetic macular oedema received Intra vitreal triamcinolone acetonide $2 \mathrm{mg}$ $(.5 \mathrm{ml})$.

\section{Inclusion Criteria}

1. Patients with Diffuse Diabetic macular oedema confirmed by Optical coherence tomography and clinical examination.

2. Patients willing to give informed consent for the procedure of intra vitreal triamcinolone. 


\section{Exclusion Criteria}

1. Eyes with macular ischemia on Fundus fluorescein Angiogram

2. Eyes with Taut posterior hyaloid or vitreo-macular traction on OCT,

3. Eyes previously undergone laser treatment or intra vitreal injection

4. Those unable to give informed consent and Unable to come for regular follow up

\section{Investigations}

When the patient presented at base line the following tests were done.

Best corrected visual acuity for distance using Snellen's chart (BCVA, UCVA)

- Slit lamp examination and biomicroscopy.

- Intra ocular pressure measurement by Goldman applanation tonometry.

- Fundus fluorescein Angiogram was done and

- Optical coherence tomography was done using spectral domain OCT to assess the central foveal thickness.

All eyes received systemic evaluation with test of $\mathrm{Hb}$, lipid profile, blood urea, serum creatinine, glycosylated haemoglobin.

Fundus fluorescein angiogram was done using Zeiss system and concentrated on the macular area noting macular ischemia and leakage. OCT was done with Spectral Domain OCT RT VUE and the average central foveal thickness was recorded. At 4 weeks OCT evaluation was reassessed for Central foveal thickness The Intra ocular pressure was recorded by Goldman Applanation tonometer The patent was followed up at 2 and 4 months On each follow up the Best corrected visual acuity was recorded for both eyes, Intra ocular pressure was measured by Goldman Applanation Tonometry, OCT recording of central foveal thickness was done

\section{Statistical Analysis}

Data is presented in percentages and described by mean \pm 1 SD. Appropriate pictorial representation is made using pie, bar, paired bar, component bar and line graphs. Kolmogrov Smirnov test for normality was employed to check if central foveal thickness followed normal distribution. Averages were compared between paired samples using paired $t$ test. A Mc Nemar Chi Square Test was performed to analyse change in vision categories within same individual. Chi Square test was employed to check for differences in proportions (CFT change Vs. Visual Acuity Change). A repeated measures analysis was done with simple contrast from baseline to 4 weeks, 2 months and 4 months central foveal thickness. A p value less than 0.05 was considered statistically significant.

\section{RESULTS}

Thirty eyes with diffuse diabetic macular oedema (DDME), of 24 patients were studied for visual outcome following intra vitreal triamcinolone were included in the study. Six (20.0\%) patients had the intervention in both eyes. The patients were aged 56.4 \pm 7.5 (Range: 45 to 73 ) years and 18 (75.0\%) were male. Duration of diabetes was $15.9 \pm 4.3$ years ranging from 10 to 29 years. Eleven (36.7\%) patients were reported to have hypercholesterolemia. After hypercholesterolemia, hypertension was the commonest $(30.0 \%)$ systemic condition reported by the patients Eight (26.7\%) patients were uncontrolled diabetic with HbA1c >7(1 with nephropathy), and 5(16.7\%) were anaemic.

\section{Assessment Pre-Intravitreal Triamcinolone Pattern as Detected through OCT}

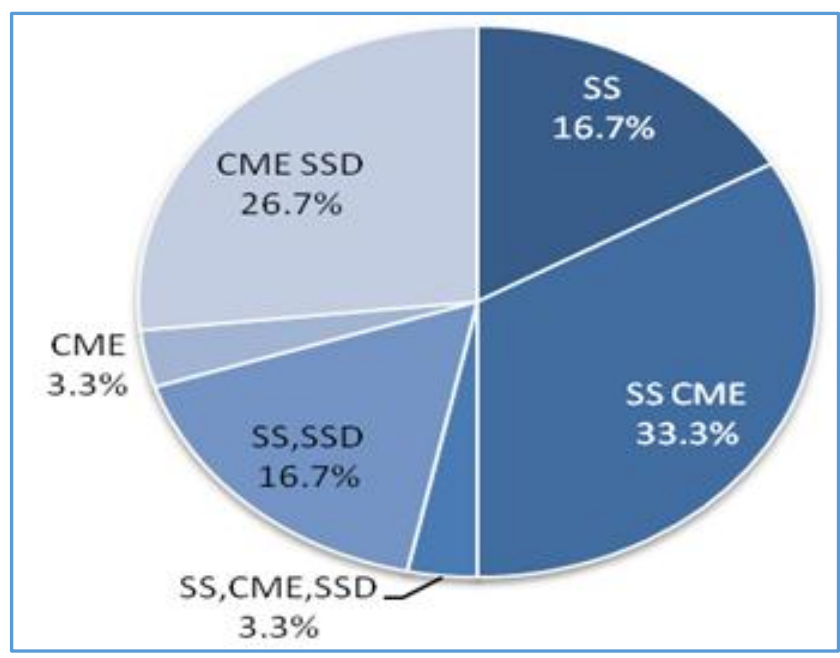

Figure 1. Pie diagram showing pattern detected through $\mathrm{OCT}$

Spongiform swelling (SS) was the commonest (21 $(70.0 \%)$ eyes) pattern as seen with OCT. Cystoid macular oedema (CME) was seen in 12 (66.6\%) eyes. Subfoveal serous detachment (SSD) was detected in 13 (46.7\%) eyes. SSD with CME was observed in 8 (26.7\%) Central Foveal Thickness. The average central foveal thickness (CFT) was $413.3 \pm 100.4$ $\mu \mathrm{m}$ ranging from 295 to $664 \mu \mathrm{m}$ in the study eye at baseline. The variable CFT followed normal distribution ( $\mathrm{p}-0.187$ ) and hence was given parametric treatment for further analysis. 


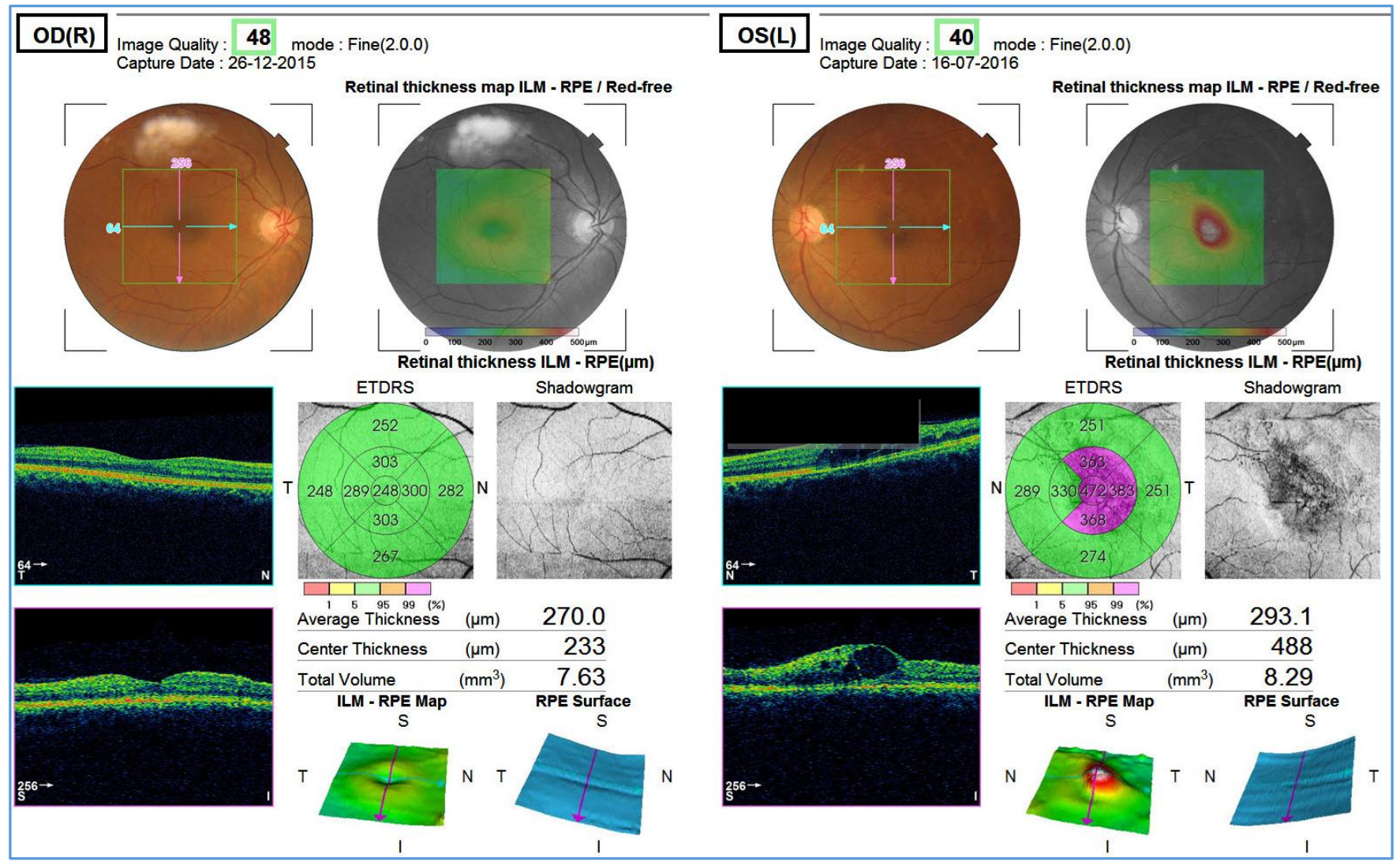

Figure 2. OCT picture showing diffused diabetic macular oedema

\section{Fellow Eye Findings}

Among the 16 unilateral DDME patients, focal CSME was seen in almost (93.8\%) all instances in the fellow eye. DDME including taut posthyaloid were seen was observed in $3(18.8 \%)$ cases and were not intervened.

Best Corrected Visual Acuity at presentation in eyes with DDME

Preoperative Visual Acuity in the Study Eye

The best corrected visual acuity (BCVA) at presentation was mostly $(60.0 \%)$ in the $6 / 36$ to $6 / 60$ category (Figure). Five (16.7\%) eyes had BCVA 6/18 to 6/24 and five had worse than $6 / 60$ in the operated eye. The BCVA fellow eye was $>=6 / 12$ in $68.8 \%$ in the unilateral cases.

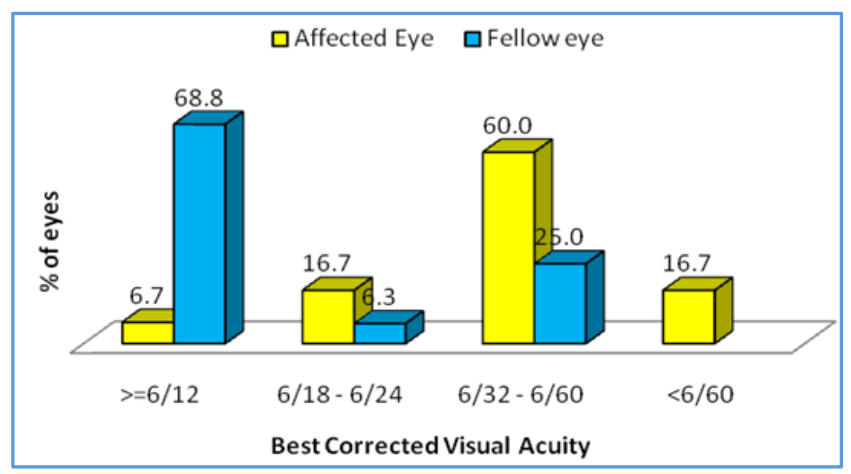

Figure 3. Paired bar chart showing pre operative best coreected visual acuity

\section{Assessment Post Intra Vitreal Triamcinolone} Central Foveal Thickness

There was $23.3 \%$ reduction in the central foveal thickness (322.2 \pm 85.3$)$ in 3 weeks post intervention when compared to the baseline $(422.0 \pm 92.1 \mu \mathrm{m})$. The thickness remained similar at 2 months $(317.4 \pm 63.8 \mu \mathrm{m})$. There was a slight $(13.1 \%)$ increase in thickness at 4 months $(358.9 \pm 119.5 \mu \mathrm{m})$ compared to the same at 2 months. The overall change in central foveal thickness at 4 months compared to the baseline was $14.5 \%$. The individual CFT difference was calculated between the baseline and at 4 months. The average of difference made in foveal thickness was $61.3 \pm 90 \mu \mathrm{m}$ [median: $75 \mu \mathrm{m}$, ranging from a $-204 \mu \mathrm{m}$ (increase), to a $220 \mu \mathrm{m}$ (decrease)].

Repeated measures analysis revealed a statistically significant $(p<0.001)$ alteration in the central foveal thickness. The contrast made from baseline visit was statistically significant at 3 weeks $(p<0.001), 2$ months $(\mathrm{p}<0.0001)$ and at 4 months ( $\mathrm{p}-0.001)$.

Figure. Changes in central foveal thickness pre and post intervention.

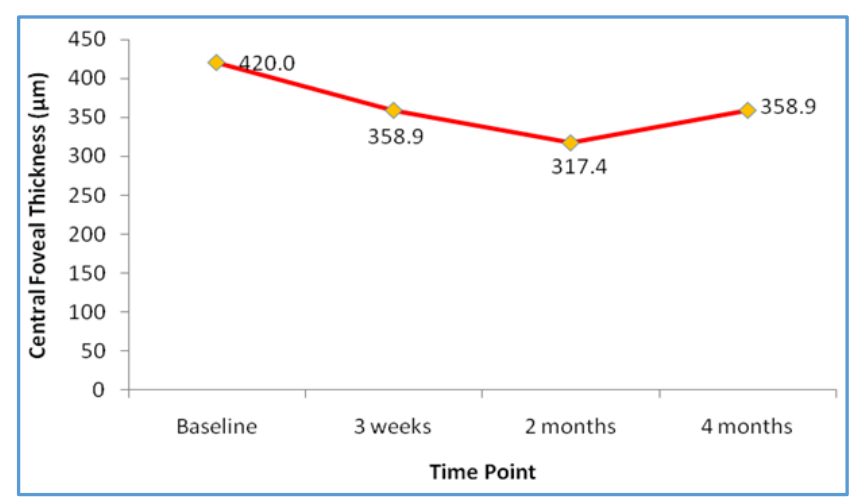

Figure 4. Graph showing central foveal thickness Change in best Corrected Visual Acuity 


\section{Three Weeks}

At three weeks, the best corrected visual acuity had improved by at least 1 line in 17(56.6\%) eyes. Four (13.3\%) eyes improved 2 lines in BCVA and there was a 4-line improvement in one eye. Best corrected vision deteriorated 1 line $(6 / 36$ at baseline to $6 / 60$ at 3 weeks) in one eye (3.3\%). Twelve (40.0\%) eyes did not show any improvement in best corrected vision.

When analysed by vision categories, there was statistically significant (p-0.016) improvement in best corrected visual acuity when compared between baseline and 3 weeks (Figure).

\section{Months}

At two months, the best corrected visual acuity had improved by at least 1 line in 22(74.3\%) eyes. Five (16.7\%) eyes improved 2 lines in BCVA and there was a 5-line improvement in (2/60 baseline to $6 / 60$ at 2 months) one eye. No change was observed in 8 (26.7\%) eyes.

When analysed by vision categories, there was statistically significant (p-0.002) improvement in best corrected visual acuity when compared between baseline and 2 months.

\section{Four Months}

At four months, the best corrected visual acuity had improved by at least 1 line in 23(76.7\%) eyes. Seven (23.3\%) eyes improved 2 lines in BCVA. No change was observed in 6 $(20.0 \%)$ eyes when compared to the baseline vision.

When analysed by vision categories, there was statistically significant $(p-<0.0001)$ improvement in best corrected visual acuity when compared between baseline and 4 months.

Changes in Best Corrected Visual Acuity

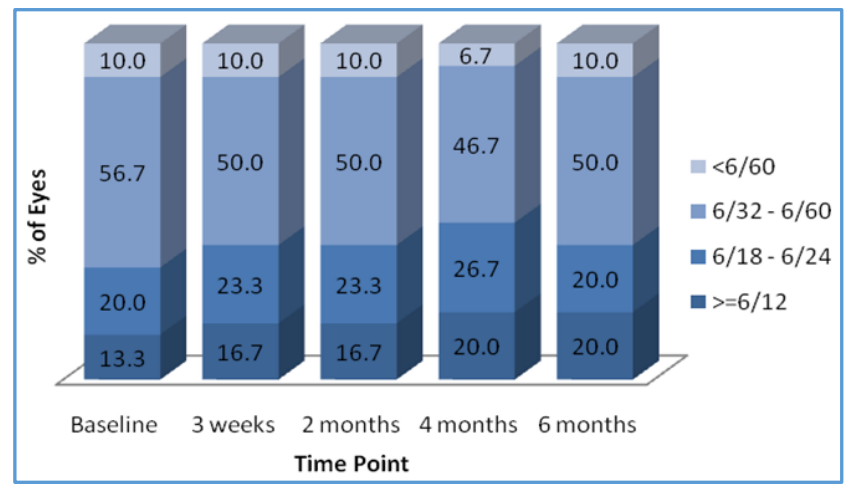

Figure 5. Bar chart showing changes in best corrected visual acuity

\section{Months}

No change was observed in best corrected visual acuity at 6 months when compared to baseline in $8(26.7 \%)$ Twenty (66.6\%) eyes showed atleast one line improvement at the end of 6 months post intervention. A reduction in vision by two lines $(6 / 36$ at baseline to $5 / 60$ at 6 months) in one eye and 4 lines (6/60 at baseline to $2 / 60$ at 6 months) in one eye.

At the end of 6 months, $12(40.0 \%)$ of the eyes had best corrected vision $6 / 24$ or better. One half of the eyes were in the $6 / 36$ to $6 / 60$ category. Three $(10.0 \%)$ eyes had vision worse than $6 / 60$ at 6 months.

\section{Doubling of the Visual Angle}

By defining the change in visual acuity based on the doubling of the visual angle criterion, the visual acuity had improved in $23.3 \%$, remained stable in $56.7 \%$ and worsened in six (20.0\%) eyes (Figure: 6) at 6 months following grid laser photocoagulation intra vitreal triamcinolone as a treatment for diffuse diabetic macular oedema.

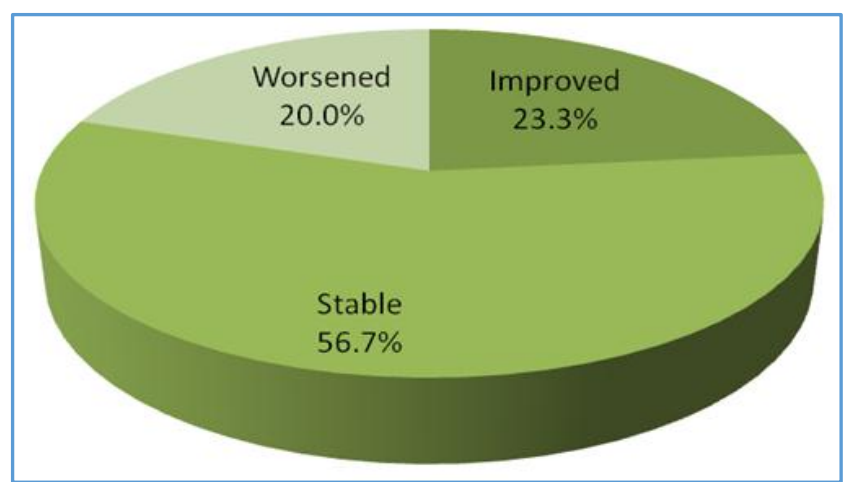

Figure 6. Change in Visual Acuity

\section{Variables associated with Change in Visual Acuity Diagnosis and Change in Visual Acuity}

The change in visual acuity was associated with the initial diagnosis made in that eye. Among eyes that had DDME improvement was seen in $29.2 \%$, stabilization in $11(45.8 \%$ ) and deterioration in $6(25.0 \%)$ eyes. Whereas all 6 eyes that had DDME with hard exudates of the fovea (HEXF), the vision did not improve because of foveal hard exudates. The difference in pattern however was not statistically significantly different (p-0.057).

Central Foveal Thickness and Change in Visual Acuity.

Figure 7 presents the change in visual acuity by CFT at 4 months. Change in visual acuity was not associated with the change that occurred in the central foveal thickness at 4 months ( $\mathrm{p}-0.648)$

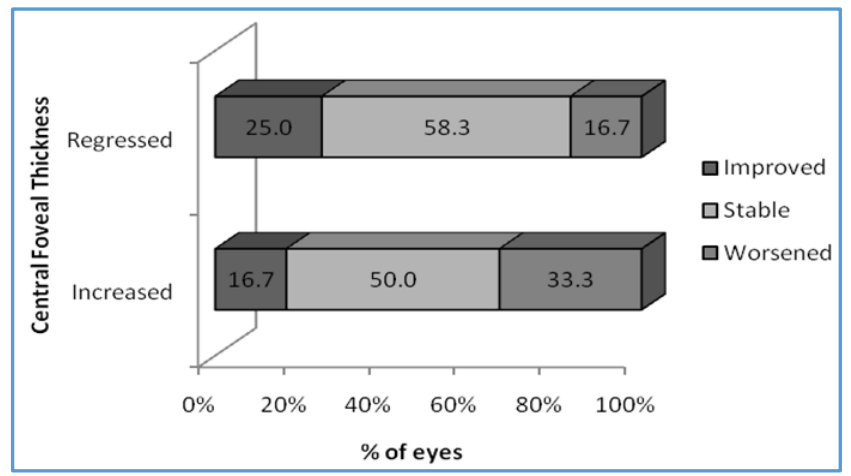

Figure 7. Central Foveal Thickness and Change in Visual Acuity

\section{Side Effects of Treatment}

In our study at 4 months 2 of the patients had cataract and rise in Intra ocular pressure was seen in 1 patient which subsided with topical antiglaucoma drugs.

\section{DISCUSSION}

Two different subtypes of diabetic macular oedema are recognized. Focal macular oedema is characterized by focal leakage from microaneurysms and is often associated with intraretinal lipid deposition in a circinate pattern. 
Diffuse macular oedema is characterized by diffuse leakage from the retinal capillaries and formation of cystoid spaces. In eyes with diffuse macular oedema, laser treatment cannot be focused on localized leaking thickening of the entire macula. In the ETDRS, grid laser treatment was applied to areas of diffuse macular oedema. Previous studies have shown, however, that eyes with diffuse macular oedema carry a particularly poor prognosis despite laser photocoagulation.

Intra vitreal triamcinolone for diabetic macular oedema DME. 3,4

Robert Machemer, MD, first advocated the use of intravitreal corticosteroid for the treatment of proliferative vitreoretinopathy. 4 Brooks McCuen, MD, and Collene Foragues demonstrated the lack of ocular toxicity of intravitreal triamcinolone acetonide in an experimental rabbit model. 5 Their results agreed with clinical observation of cases in which corticosteroids were accidentally injected into the eye, and no major toxic reactions were detected. For the past several decades since that time, intravitreal corticosteroids have been used in limited trials in patients without evidence of ocular toxicity.

Triamcinolone acetonide 5 is a corticosteroid suspension that has been used in periocular injections ${ }^{6}$ for the treatment of-

- Cystoid macular oedema secondary to uveitis and as a result of intraocular surgery.

- Uveitic cystoids macular oedema,

- Exudative age related macular degeneration.

- Neovascular glaucoma.

- Proliferative diabetic retinopathy.7

- Hypotony.

- Proliferative vitreoretinopathy.

- Macular oedema secondary to retinal vascular occlusive disease.

- Pseudophakic cystoid macular oedema.

- Cystoid macular oedema in retinitis pigmentosa. IVTA has also been reported to have favourable results in the treatment of diffuse diabetic macular oedema. The exact mechanism of action of corticosteroids in the treatment of macular oedema is unknown. However, the rationale behind their use lies in their ability to inhibit the arachidonic acid pathway, of which prostaglandin is a product. Corticosteroids may also downregulate the production of vascular endothelial growth factor (VEGF) ${ }^{9}$.

- Another potent steroid dexamethasone implant is being used in the management of diabetic macular oedema. preliminary studies have shown on dramatic resolution of macular oedema and foveal thickness.

\section{CONCLUSION}

There was statistically significant difference in the central foveal thickness and also statistically significant improvement in visual acuity was observed.

\section{REFERENCES}

[1] Pulido JS. Diabetic retinopathy in the new millennium. Ophthalmology clinics of North America 1999;12(4):563-71.

[2] Photocoagulation for diabetic macular edema: early treatment of diabetic retinopathy study report no 4 . The early treatment diabetic retinopathy study research group. International ophthalmology clinics 1987;27(4):265-72.

[3] Massin P, Aurdren F, Haouchin B, et al. Intravitreal triamcinolone acetonide for diabetic diffuse macular edema: preliminary results of a prospective controlled trial. Ophthalmology 2004;111(2):218-24.

[4] Vedantham V, Kim R. Intravitreal injection of triamcinolone acetonide for diabetic macular edema: principles and practice. Indian J Ophthalmol 2006;54(2):133-7.

[5] Thompson MJ, Michel SIP. Diabetic macular edema: a review of presentpast and future therapies. Intrnational Ophthalmology clinics 2004;44(4):51-67.

[6] Vedantham V. Periocular corticosteroid therapy: comments. Br J Ophthalmol 2004;88(5):724-5.

[7] Mcdonald HR, Schatz H. Visual loss following panretinal photocoagulation for proliferative diabetic retinopathy. Ophthalmology 1985;92(3):385-93.

[8] Martidis A, Duker JS, Greenberg PB, et al. Intravitreal triamcinolone for refractory diabetic macular edema. Ophthalmology 2002;109(5):920-7.

[9] Jonas JB, Kreissig I, Sofker A, et al. Intravitreal injection of triamcinolone for diffuse diabetic macular edema. Arch Ophthalmol 2003;121(1):57-61. 